\title{
Molecular Interaction between Colorimetric Sensor Array and Volatile Organic Compounds Considering Density Functional Theory
}

\author{
Haiyang Gu, ${ }^{1,2}$ Yanhui Sun, ${ }^{1}$ Xingyi Huang, ${ }^{2 *}$ and Huang Dai ${ }^{3}$ \\ ${ }^{1}$ School of Bio Food Engineering, Chuzhou University, Chuzhou 239000, China \\ ${ }^{2}$ School of Food and Biological Engineering, Jiangsu University, Zhenjiang 212013, China \\ ${ }^{3}$ College of Biosystems Engineering and Food Science, Zhejiang University, Hangzhou 310058, China
}

(Received May 19, 2016; accepted June 14, 2016)

Keywords: colorimetric sensor array, density functional theory, copper porphyrin, food quality

A theoretical study based on density functional theory (DFT) at the B3LYP/LANL2DZ level was carried out to investigate the ability of a colorimetric sensor array (CSA) to bind volatile organic compounds (VOCs). Copper porphyrin (CuP) is the common dye that is used to represent a CSA in this study. There are three possible spin states for CuP and CuP-VOCs, namely, singlet, triplet, and quintet. However, the ground state for $\mathrm{CuP}$ is the triplet state, except for $\mathrm{CuP}-\mathrm{O}_{2}$. This theoretical study reflects that $\mathrm{CuP}$ is very sensitive to $\mathrm{N}_{2}$ necessitating a change of the carrier gas from $\mathrm{N}_{2}$ to another gas. The ability of CuP to bind VOCs can be classified into three levels on the basis of binding energy. The results of this study suggest that theoretical investigations will be useful for optimizing the CSA sensor for a specific analyte.

\section{Introduction}

The evaluation of food quality is very important to consumers and the food industry.(1) A consumer may feel uncomfortable when the food quality decreases during storage, and may even suffer from physical symptoms. However, it is difficult to address the status of food quality until the food has spoiled. ${ }^{(2)}$ Food odors, which are produced by bacteria, are always used to evaluate food freshness. According to previous studies, gas chromatography (GC) and the electronic nose (EN) have been employed for the detection of volatile organic compounds (VOCs). These previous methods are based on weak molecular interactions, such as those of VOCs absorbed into conductive polymers or onto metal oxide surfaces. Thus, the prior technologies are considered to have poor sensitivity, poor discrimination, high energy consumption, expensive testing fees, and susceptibility to the relative humidity of an analyte. It is difficult to accomplish the rapid and sensitive evaluation of food quality required by food manufacturers and salesmen.

In the past decade, a novel method, the colorimetric sensor array (CSA) method, has been developed to detect VOCs. It is composed of a series of cross-reactive dyes printed on hydrophobic film. In contrast with traditional detection methods for VOCs, CSA is a high sensitivity, high discrimination and anti-interference method. For a 24-sensor array, 1012 to 25672 distinct patterns of CSA can be recognized. ${ }^{(3)}$ The traditional detection method for VOCs is always affected by

"Corresponding author: e-mail: 1323992481@gg.com

http://dx.doi.org/10.18494/SAM.2017.1406 
changes in humidity. CSA is an ideal method that is essentially impervious to changes in relative humidity from 2000 to $20000 \mathrm{ppm}$ in the environment. ${ }^{(3)}$ High sensitivities (ppb) have been proved both theoretically and experimentally (i.e., using amines, carboxylicacids, and thiols). ${ }^{(4)}$ Thus, the CSA method has emerged as a powerful method for the analysis and evaluation of food quality and safety, which can produce a unique composite response for each sample. This helpful sensor mimics the mammalian olfactory system based on strong molecular interactions. The CSA method has been proved to be very useful for the evaluation of food quality, such as fish freshness, ${ }^{(5)}$ soft drink flavor, ${ }^{(6)}$ bacteria, ${ }^{(7)}$ and artificial sweetener. ${ }^{(8)}$

However, the CSA method is a novel detection method where the sensor design is based on trial and error. It will require much money and time to design a proper CSA sensor for a specific analyte. In this study, we investigated the molecular interaction between the CSA sensor and VOCs. Copper porphyrin $(\mathrm{CuP})$ is a lewis acid/base dye that is very sensitive to VOCs, and is always used in the CSA sensor. Thus, the CSA sensor is represented by CuP. Molecular interaction between the CSA sensor and VOCs is employed in the framework of the density functional theory (DFT) at B3LYP/LANL2DZ level. This calculation method has been proved to be suitable for studying the interaction between metal porphyrin and VOCs.

This study, therefore, had the aim of devising a simple CSA sensor design based on the theoretical study.

\section{Colorimetric Sensor Array and Computational Method}

\subsection{Colorimetric sensor array}

A schematic of the experimental apparatus is shown in Fig. 1. The CSA sensor consisted of dyes that were printed on $\mathrm{C} 2$ reverse phase silica gel plates. ${ }^{(5)}$ There are two processes in the reaction of the CSA sensor with VOCs. First, the dyes on the CSA sensor bind the VOCs that are emitted from food or beverages during storage. The molecular interaction between the CSA sensor and VOCs is shown in Fig. 1, and all the other molecular interactions are similar to it. Second, the result of reaction between the CSA sensor and VOCs is displayed as a difference image. The difference image shown in Fig. 1 was obtained by subtracting the post-reaction image from the pre-reacation image (i.e., post-reaction on red, green, and blue values subtracted from pre-reaction red, green, and blue values).

Then, RGB data from difference images are analyzed by pattern recognition using, for example, a support vector machine, neural networks, and partial least squares regression.

\subsection{Computational method}

The CSA sensor model used is represented by a simple copper-porphyrin molecule, $\mathrm{CuP}$ and $\mathrm{CuP}-\mathrm{VOCs}$, without any meso substituent. All calculated models for each $\mathrm{CuP}$ and VOC have been obtained from the Cambridge Crystallographic Data Centre (CCDC). The CuP-VOCs combination is constructed by adding VOCs above the metal porphyrin plane defined by the four nitrogen $(\mathrm{N})$ atoms of the metalporphyrin molecule. The VOC-Cu angle is about $135^{\circ}$, which is consistent with that in a previous study. ${ }^{(9)}$ The interatomic distance between the metalporphyrin plane and each VOC is from 3-4 $\AA$, which is consistent with a previous study. ${ }^{(10)}$ All models were calculated using DFT at the B3LYP/LANL2DZ level. Metalporphyrin and similar complexes are shown to be 


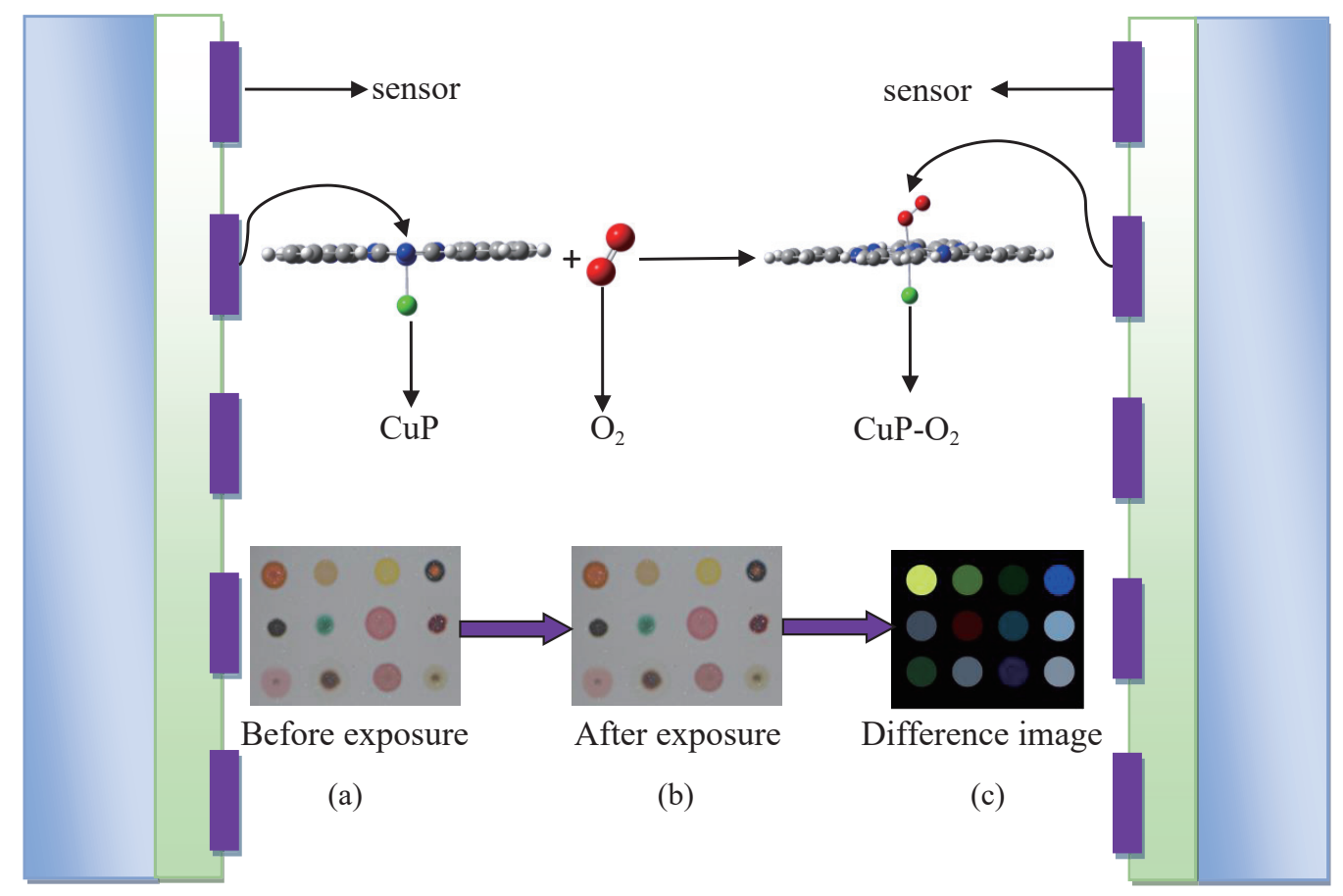

Fig. 1. (Color online) Schematic of experimental apparatus consisting of C2 reverse phase silica gel plate and dyes printed on it. A difference image is obtained by subtracting the pre-exposure image from the post-exposure image.

highly suitable sensors by DFT-based methods. ${ }^{(11,12)}$ To avoid any shortcomings, the initial structure was optimized at one of three possible spin states (i.e., singlet, triplet and quintet). Molecule optimization for each initial model was carried out by identifying the most stable structure by comparing relative energies. Further theoretical analysis is performed on the basis of the ground state structure. All calculations were carried out using the Gaussian 09 program package.

\section{Results and Discussion}

\subsection{Relative energy}

As is shown in Table 1, the relative energies for all models are calculated at three possible spin states. For unligated $\mathrm{CuP}$, the system in the triplet state is much more stable than those in singlet and quintet states. The energy gaps for systems in the singlet and quintet states relative to the triplet state are 19.204 and $56.130 \mathrm{kcal} / \mathrm{mol}$, respectively. However, for CuP-VOCs, the triplet state is the most stable, except for $\mathrm{CuP}-\mathrm{O}_{2}$. Jensen and Ryde ${ }^{(13)}$ stated that the smaller energy gap indicates that the process of $\mathrm{CuP}$ binding with VOCs probably occurs through more than one pathway, making the binding process more efficient and faster. It is interesting to see that $\mathrm{CuP}-\mathrm{L} 3$ possesses the highest relative energy, followed by CuP- $\mathrm{N}_{2}$ and CuP-L5, whereas $\mathrm{CuP}_{-} \mathrm{O}_{2}, \mathrm{CuP}-\mathrm{H}_{2} \mathrm{~S}$, and CuP-L1,2,4 possess low relative energies. This result indicates that the binding ability of CuP with VOCs is not only represented by binding energy but also affected by relative energy. This idea will be used to 
Table 1

Relative energies for all CuP-VOCs and CuP relative to the most stable one (given in $\mathrm{kcal} / \mathrm{mol}$ ). L1 is trimethylamine, L2 is propanol, L3 is propane, L4 is ethyl acetate, L5 is butanone, and L6 is propionaldehyde.

\begin{tabular}{lccc}
\hline Complex & $\Delta$ singlet & $\Delta$ triplet & $\Delta$ quintet \\
\hline $\mathrm{CuP}$ & 19.204 & 0 & 56.130 \\
$\mathrm{CuP} \mathrm{O}_{2}$ & 66.674 & 20.058 & 0 \\
$\mathrm{CuP}-\mathrm{N}_{2}$ & 39.584 & 0 & 63.623 \\
$\mathrm{CuP}_{2} \mathrm{~S}$ & 19.178 & 0 & 55.378 \\
$\mathrm{CuP}-\mathrm{L} 1$ & 18.818 & 0 & 62.693 \\
$\mathrm{CuP}-\mathrm{L} 2$ & 19.589 & 0 & 56.940 \\
$\mathrm{CuP}-\mathrm{L} 3$ & 44.356 & 0 & 56.303 \\
$\mathrm{CuP}-\mathrm{L} 4$ & 19.329 & 0 & 59.208 \\
$\mathrm{CuP}-\mathrm{L} 5$ & 29.004 & 0 & 59.115 \\
\hline
\end{tabular}

evaluate the binding ability of $\mathrm{CuP}$ with VOCs, as described below, when $\mathrm{CuP}$ possesses the same or very close binding energies for two VOCs.

\subsection{Molecular structure}

The initial geometric structures for $\mathrm{CuP}$ and CuP-VOCs were obtained from CCDC. The axial ligand is a chlorine atom coordinated with the $\mathrm{Cu}$ atom in the center of the metalporphyrin plane, as always used in the CSA sensor. The initial manner of binding is an important factor in the computational result. End-on bent and side-on bent are considered to be the binding geometries for $\mathrm{CuP}$ with VOCs. However, the end-on bent geometry is considered to be the most stable, as proved by the theoretical study and X-ray structure analysis. Thus, the initial binding geometry is designed to be end-on bent. To avoid any shortcomings, the optimization of the initial geometric structure was carried out for three different spin states (low-spin state, intermediate-spin state, and highspin state). As is shown in Fig. 2, three important geometric structures are displayed for the three different spin states. Note that the distance of $\mathrm{Cu}$ atom displacement from the metalloporphyrin plane is much larger than the distance between the $\mathrm{Cu}$ atom and $\mathrm{Cl}$, and $\mathrm{VOC}$ atoms. This result indicates that the influence of VOCs on the metalloporphyrin plane is via the $\mathrm{Cu}$ atom to other atoms.

\subsection{Charge distribution}

As shown in Fig. 3, the charge distributions between the $\mathrm{N}$ and $\mathrm{Cu}$ atoms in the metalloporphyrin plane display a strong linear relationship. From this figure, it is clear that a larger positive charge on the $\mathrm{Cu}$ atom will cause the $\mathrm{N}$ atom to be more negative. CuP-L1 possesses the smallest positive charge of the $\mathrm{Cu}$ atom, and smallest negative charge of the $\mathrm{N}$ atom at the top left of the plot. On the other hand, $\mathrm{CuP}, \mathrm{CuP}-\mathrm{H}_{2} \mathrm{~S}$, and $\mathrm{CuP}-\mathrm{L} 3$ possess larger positive charge and larger negative charge on the $\mathrm{Cu}$ atom and the $\mathrm{N}$ atom, respectively, as shown at the right lower corner of the plot. From the line in Fig. 3, note that binding with VOCs makes the $\mathrm{Cu}$ atom more negative and the $\mathrm{N}$ atom more positive. The reason for this may be that the sharing of an electron through the efficient backbonding interaction between the $\mathrm{Cu}$ atom and VOCs will change the charge distribution on $\mathrm{Ag}$ and $\mathrm{N}$ atoms. Besides the influence of electron transfer, the charge distribution also reflects that VOCs affect the CSA sensor through the electron exchange transfer from the VOCs to the porphyrin plane. 


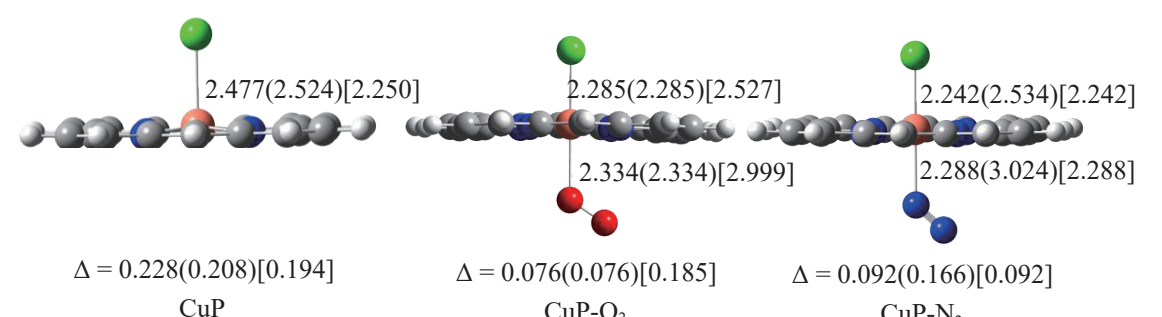

(a)

(b)

(c)

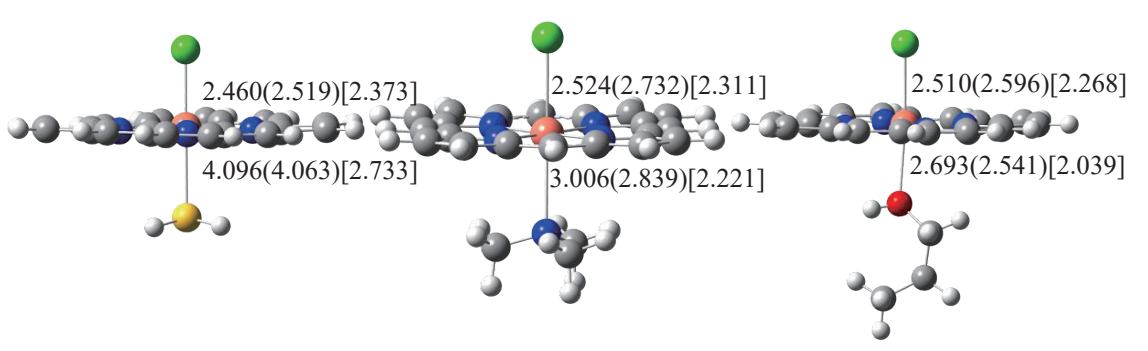

$\Delta=0.228(0.201)[0.109]$
CuP- $\mathrm{H}_{2} \mathrm{~S}$

(d)
$\Delta=0.130(0.072)[0.037]$

CuP-L1

(e)
$\Delta=0.108(0.109)[0.160]$

CuP-L2

(f)

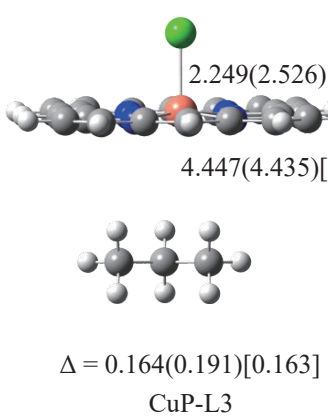

(g)
2

$2.520(2.583)[2.264]$
$2.365(2.584)[2.267]$

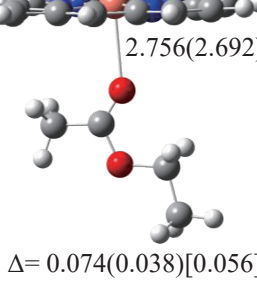

CuP-L4

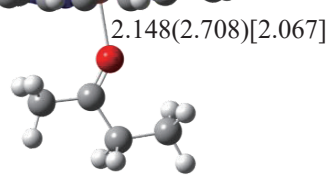

$\Delta=0.081(0.047)[0.082]$ CuP-L5

Fig. 2. (Color online) Optimized structures for CuP-VOCs at three different spin states. $\Delta$ is the displacement of a $\mathrm{Cu}$ atom out of the metalloporphyrin plane. Data for CuP-VOCs in the singlet state is shown without parentheses, in parentheses for the triplet state, and in brackets for the quintet state.

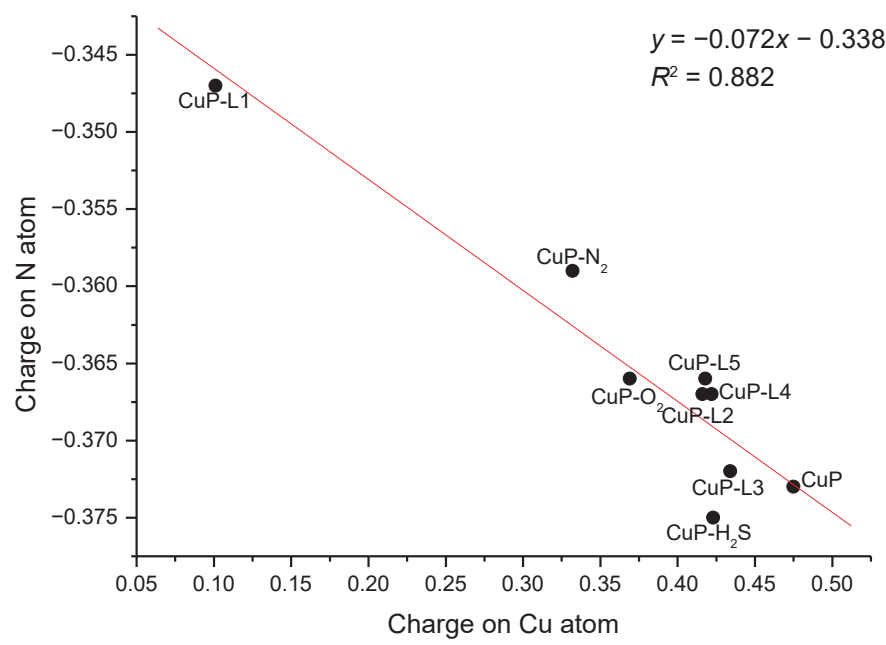

Fig. 3. (Color online) Relationship between charges on nitrogen atom and $\mathrm{Cu}$ atom for $\mathrm{CuP}$ and its complexes coordinated with different VOCs. 


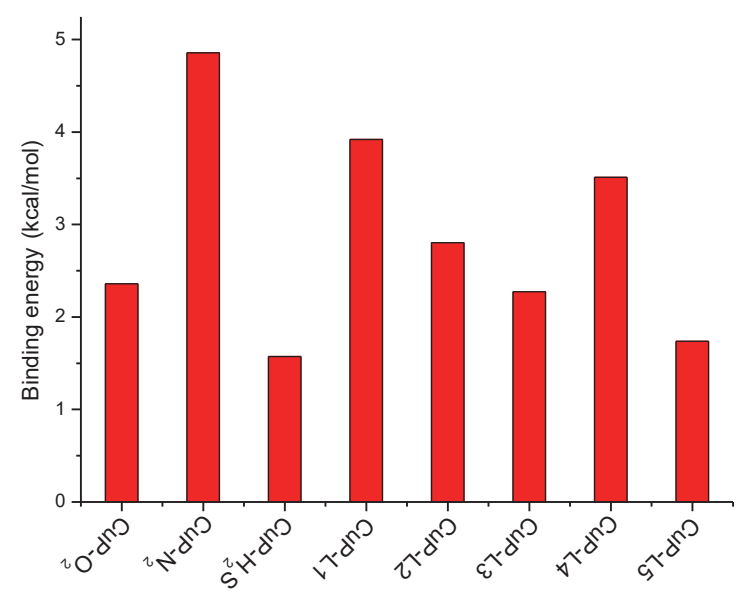

Fig. 4. (Color online) Binding energies (given in $\mathrm{kcal} / \mathrm{mol}$ ) for each $\mathrm{CuP}-\mathrm{VOC}$ obtained by subtracting the prebinding energy from the post-binding energy based on the most stable state.

\subsection{Binding energy}

As was found in our previous study, the binding process of metalloporphyrin with VOCs can be described as $\mathrm{CuP}+\mathrm{VOCs} \rightarrow \mathrm{CuP}-\mathrm{VOCs}$. This metalloporphyrin model will absorb or release energy following the above process. The energy change corresponds to the binding energy. The binding energy, which shows the energy change in each model, was obtained by subtracting the pre-binding energy from the post-binding energy. The detailed relationship can be written as: $B E$ $=E_{\mathrm{O}_{2}}+E_{\mathrm{CuP}}-E_{\mathrm{O}_{2}-\text { Cup. }}$. Figure 4 shows the binding energy for each CuP-VOC. Note that CuP-N ${ }_{2}$ possesses the largest binding energy, followed by CuP-L1,2,4, whereas $\mathrm{CuP}-\mathrm{O}_{2}, \mathrm{CuP}-\mathrm{H}_{2} \mathrm{~S}$, and $\mathrm{CuP}-\mathrm{L} 3,5$ possess relatively low binding energies. This result indicates that $\mathrm{CuP}$ is very sensitive to $\mathrm{N}_{2}$. However, $\mathrm{N}_{2}$ is always used to carry the analyte to the reaction chamber. Thus, we must change the carrier gas when using the CSA sensor containing CuP dye. VOCs containing $\mathrm{N}$ atoms are always used as indicators of food freshness. ${ }^{(14)}$ In this study, CuP possesses the highest binding energy for L1, which means that this dye is very sensitive to VOCs containing $\mathrm{N}$ atoms. Hence, the CSA sensor containing CuP dye will be useful for the evaluation of food freshness. L2, L3, L4, and L5 are always used to evaluate the change in food quality during storage. From the above results of binding energy, it is clear that the CSA sensor containing $\mathrm{CuP}$ is most sensitive to $\mathrm{L} 4$, followed by L2 and L3, and L5 incites the last sensitivity.

\section{Conclusions}

We provided theoretical evidence that the binding ability of CSA sensors changes for different VOCs. The binding ability of the CSA sensor with different VOCs can be used to select a sensor for constructing a suitable CSA system for evaluating food quality. A series of theoretical computations were performed to investigate the property of CSA sensors by the DFT method at the B3LYP/ LANL2DZ level. First, the most stable structure for each model was determined by comparing the relative energies in three different possible states. Note that almost all $\mathrm{CuP}$ and $\mathrm{CuP}-\mathrm{VOCs}$ possess the most stable structure in the triplet state, except for $\mathrm{CuP}-\mathrm{O}_{2}$. The analysis of charge distribution 
indicates that the nature of the influence of VOCs on the metalloporphyrin plane is electron transfer from the $\mathrm{Cu}$ atom to the metalporphyrin plane. Finally, the analysis of binding energy shows that CuP- $\mathrm{N}_{2}$ possesses the largest binding energy, followed by CuP-L1,2,4 and $\mathrm{O}_{2}$, whereas CuP-L3,5 and $\mathrm{CuP}-\mathrm{H}_{2} \mathrm{~S}$ possess relatively smaller binding energies. This theoretical study may also be useful for other metalloporphyrins. Further research is needed to optimize the CSA sensor on the basis of our results for specific foods to expand the range of application of CSA technology.

\section{Acknowledgements}

This study was sponsored by the Scientific Research Foundation of Chuzhou University (Project No. 2014qd042); Planning Project for Jianghuai Watershed Coordination Center (Project No. 2014GH37); Anhui Research Center for Heat-Sensitive Material Open Fund (Project No. 2015RMY04); Horizontal Subject (Project No. HX201558); Anhui Natural Science Foundation (Project No.1608085MC73); and Anhui Province Public Welfare Research Project (Project No.1604f0704051).

\section{References}

1 K. Aronsson, M. Lindgren, B. R. Johansson, and U. Rönner: Innovative Food Sci. Emerg. Technol. 2 (2001) 41.

2 M. Wandel and A. Bugge: Food Qual. Preference 8 (1997) 19.

3 K. S. Suslick, N. A. Rakow, and A. Sen: Tetrahedron 60 (2004) 11133.

4 K. S. Suslick, N. A. Rakow, M. E. Kosal, W. B. McNamara III, and A. Sen: Proc. ISOEN. 2 (2003) 46.

5 X. Huang, J. Xin, and J. Zhao: J. Food Eng. 105 (2011) 632.

6 C. Zhang and K. S. Suslick: J. Agric. Food Chem. 55 (2007) 237.

7 J. R. Carey, K. S. Suslick, K. I. Hulkower, J. A. Imlay, K. R. Imlay, C. K. Ingison, J. B. Ponder, A. Sen, and A. E. Wittrig: J. Am. Chem. Soc. 133 (2011) 7571.

8 C. J. Musto, S. H. Lim, and K. S. Suslick: Anal. Chem. 81 (2009) 6526.

9 A. Abdurahman and T. Renger: J. Phys. Chem. A 113 (2009) 9202.

10 B. Minaev: Spectrochim. Acta, Part A 60 (2004) 3213.

11 W. Nam, S.-Y. Oh, Y. J. Sun, J. Kim, W.-K. Kim, S. K. Woo, and W. Shin: J. Org. Chem. 68 (2003) 7903.

12 J. Yang and P. Huang: Chem. Mater. 12 (2000) 2693.

13 K. P. Jensen and U. Ryde: J. Biol. Chem. 279 (2004) 14561.

14 J. Botta, J. Lauder, and M. Jewer: J. Food Sci. 49 (1984) 734. 